\title{
Atuação do profissional de Educação Física em sala de pré-parto hospitalar: interfaces com equipe multiprofissional
}

\author{
Physical Education professional performance in the hospital pre-delivery room: \\ interfaces with a multidisciplinary team \\ Juliana Quadros Santos Rocha1, Lidiane Pozza Costa², Marcelo Zanusso Costa², Fernanda Grill da Silva, \\ Andressa da Silva Arduim4, Daiana Carvalho Borges ${ }^{1}$, Mariângela da Rosa Afonso ${ }^{3}$, \\ Fernanda de Souza-Teixeira ${ }^{3}$ \\ ${ }^{1}$ Universidade Federal do Rio Grande (FURG), Rio Grande, Brasil \\ 2 Hospital Escola da Universidade Federal de Pelotas (HE/UFPel), Pelotas, Brasil \\ 3 Universidade Federal de Pelotas (UFPel), Pelotas, Brasil \\ ${ }^{4}$ Universidade Federal do Rio Grande do Sul (UFRGS), Porto Alegre, Brasil
}

\section{HISTÓRICO DO ARTIGO \\ Recebido: 21 setembro 2021 Revisado: 25 outubro 2021}

Aprovado: 03 novembro 2021

\section{PALAVRAS-CHAVE:}

Educação Física; Equipe

Multiprofissional; Parto

Humanizado.

\section{KEYWORDS:}

Physical education and training; Patient care team; Humanizing delivery.

\section{PUBLICAD0:}

01 janeiro 2022

\section{RESUMO}

INTRODUÇÃO: A atuação dos profissionais de Educação Física (PEF) em ambiente hospitalar ainda carece de ser conhecida e reconhecida.

OBJETIVO: O objetivo do estudo é descrever as intervenções realizadas por PEF em sala de préparto hospitalar (SPPH) e a percepção dos demais profissionais de saúde sobre sua participação na equipe multiprofissional.

MÉTODOS: Trata-se de um estudo descritivo e transversal, a partir de registros hospitalares de atendimentos a parturientes, no período de março de 2016 a agosto de 2017; e entrevistas com outros profissionais de saúde atuantes em SPPH. Os dados foram analisados por frequências relativas e absolutas e mediante análise de conteúdo.

RESULTADOS: O PEF participou do atendimento a 59 parturientes nesse período, utilizando prioritariamente técnicas combinadas com movimentação pélvica em bola suíça, caminhada e banho de aspersão. Oito foram os profissionais entrevistados, descrevendo uma contribuição positiva à equipe pelos PEFs.

CONCLUSÃO: Há reconhecimento aos PEF tanto pelo alívio da dor às parturientes e aceitação do tratamento, quanto pela contribuição deste profissional à equipe multiprofissional.

\section{ABSTRACT}

BACKGROUND: Physical Education Professionals (PEP) performance in hospital attendance still needs to be known and recognized.

OBJECTIVE: The purpose of this study is to describe the interventions performed in the hospital pre-delivery room by PEP and the perception of other health professionals about their performance.

METHODS: Descriptive and cross-sectional study. Clinical records, from March 2016 to August 2017, were consulted; and interviews with professionals working in the pre-delivery room were done. Descriptive analyses and a content analysis were performed.

RESULTS: PEP participated in the multidisciplinary care of 59 parturient women. Combined techniques were predominantly used, involving mostly: walking, pelvic movement with the use of a Swiss ball and a sprinkle bath. Eight interviews were carried out and it was possible to verify a positive contribution of the PEP.

CONCLUSION: PEF contributed to the relief of pain of the parturient and that the presence of a PEP contributed to the better acceptance of the interventions performed by nursing team. 


\section{INTRODUÇÃo}

A Educação Física teve uma inserção gradual na área da saúde, sendo reconhecida oficialmente neste campo de atuação em 1998, com a resolução $n^{\circ} 2871$ (BRASIL, 2003), visto que anteriormente estava associada, prioritariamente, às escolas como componente curricular. Desde sua regulamentação, a inclusão da área em novas políticas e/ou iniciativas no campo da saúde pode ser observada, por exemplo, na Política Nacional de Promoção da Saúde e entre as possíveis profissões formadoras dos Núcleos de Apoio à Saúde da Família (BRASIL, 2006; 2008). O profissional de educação física (PEF) se articula ao ideário de promoção da saúde e sua participação é considerada nas equipes de saúde como forma de ampliação de cuidado no processo saúde-doença, justificada pelas mudanças na saúde populacional causadas pelas transições epidemiológica, nutricional e demográfica (SCABAR et al., 2012; CARVALHO, 2016; FERREIRA; FERREIRA, 2017). Recentemente a sua atuação em ambiente hospitalar foi afiançada pela Resolução 391/2020 explicitando as competências e atribuições nesse âmbito (STEINHILBER, 2020).

Os hospitais são considerados instituição complexa, com densidade tecnológica específica, de caráter multiprofissional e interdisciplinar, responsável pela assistência aos usuários com condições agudas ou crônicas, que apresentam potencial complicação de seu estado de saúde, exigindo-se assistência contínua em regime de internação e ações que abrangem a promoção da saúde, a prevenção de agravos, o diagnóstico, o tratamento e a reabilitação (BRASIL, 2013). Neste ínterim, são espaços importantes para a atuação dos PEFs já que a prática de atividade física tem sido amplamente utilizada como estratégia não medicamentosa para o tratamento de doenças, a manutenção da saúde e a prevenção de perdas e acometimentos à saúde em decorrência do repouso, da inatividade física ou do sedentarismo (COELHO; BURINI, 2009), tanto para a população em geral como para aquelas pessoas que necessitam atendimento especializado como é o caso das gestantes (OMS, 2020).

Referente à gestação, entendida como fenômeno fisiológico que envolve mudanças dinâmicas do ponto de vista físico, social e emocional (LIMA et al., 2019; ALVES et al., 2020) a atuação do PEF é reconhecida na estimulação de diversas capacidades físicas (força muscular, flexibilidade, capacidade cardiorrespiratória, equilíbrio) (LOGAN, 2016), na preparação para o parto e na contribuição de um período pós-parto saudável (OMS, 2020). No entanto, pouco se conhece sobre a atuação do PEF com gestantes internadas e em concreto em sala de pré-parto hospitalar (SPPH). De fato, não foram encontrados artigos científicos que tratem da temática.

Considerando que o trabalho de parto (TP) é um momento significativo na vida da mulher e da família, com repercussões sobre a saúde da criança (RAMIRES et al., 2014; SCABAR et al., 2012) que pode ter repercussões sobre o desenvolvimento infantil, o parto humanizado é enaltecido pelo Ministério da Saúde (MS), preconizando o parto por via vaginal, respeitando a individualidade da mulher, garantindo o direito ao nascimento sadio e harmonioso do bebê e auxiliando no processo de formação ou transformação da família (LOGAN, 2016). Isso devido a que o parto por via vaginal favorece o contato pele a pele, o clampeamento tardio do cordão umbilical, a amamentação precoce, um menor risco de infecções e menor tempo de internação (BRASIL, 2000). Contudo, o parto vaginal é considerado um processo doloroso que envolve aspectos biológicos, culturais, socioeconômicos e emocionais - que podem afetar a percepção da parturiente frente a este rocesso (PIMENTEL; OLIVEIRA FILHO, 2016; SPERLING et al., 2016). Assim, a equipe multiprofissional que atua na assistência ao parto objetiva diminuir estressores e possíveis despreparos enfrentados pela mulher, preconizando a busca pelo alívio da dor e do desconforto e a autonomia da mulher como protagonista no processo de parturição (MAFETONI; SHIMO, 2016).

Neste ínterim, o MS recomenda a oferta dos métodos não farmacológicos para alívio de dor para as parturientes no processo de parto sendo eles: as caminhadas, as modificações de postura, as massagens, o uso da bola suíça e o banho de aspersão entre outros, utilizados de forma isolada (somente um método aplicado à parturiente) ou combinada (uso de dois ou mais métodos de forma concomitante (BRASIL, 2017). Apesar dos exercícios que compõem os métodos não farmacológicos serem de conhecimento do PEF, encontramos apenas relato de sua atuação neste ambiente (MELO; DALLA DÉA, 2013).

Considerando ainda que, desde 2011 a Empresa Brasileira de Serviços Hospitalares - EBSERH (EBSERH, 2020) contempla os PEFs como profissionais de saúde de seu quadro, que em um dos hospitais universitários do sul do Brasil, desde 2016, o PEF faz parte da equipe multiprofissional que atua na ala de obstetrícia e em SPPH, e que nesse hospital têm residentes das áreas de Educação Física, Nutrição e Odontologia vinculados ao programa de residência multiprofissional em atenção à saúde da criança, prestando atendimentos à crianças e gestantes nele internadas, conhecer e compartilhar as experiências e práticas profissionais é de fundamental relevância.

Pelo que, o objetivo deste estudo foi descrever as atuações do PEF, em SPPH, junto as parturientes; bem como, conhecer a percepção dos demais profissionais de saúde sobre sua atuação nos atendimentos da equipe multiprofissional nesse cenário.

\section{MÉTODOS}

Trata-se de um estudo descritivo com abordagens quantitativa e qualitativa, realizado a partir de prontuários de gestantes atendidas por equipe multiprofissional em SPPH com a presença de PEF, durante o período de março de 2016 à agosto de 2017, e mediante entrevista com os demais profissionais de saúde que atuam em SPPH, sobre a percepção da presença do PEF como membro da equipe multiprofissional e de sua atuação com o uso dos métodos não farmacológicos. A SPPH está inserida no setor de obstetrícia de um hospital universitário do sul do Brasil. Este hospital presta atendimento a 28 municípios da região, exclusivamente pelo Sistema Único de Saúde, representando uma estrutura de saúde de referência para o município e macrorregião. Além disso, os serviços aderem à Política Nacional de Humanização, o que torna as práticas humanizadas atividades rotineiras dentro da instituição. A SPPH é composta por dois leitos, um banheiro com chuveiro, um espaldar e duas bolas suíças para aplicação dos métodos 
não farmacológicos para alívio de dor. O setor possui ainda vinte leitos de internação disponíveis. A equipe multiprofissional que compõe SPPH são médicos obstetras, enfermeiros obstetras e técnicos em enfermagem. Os PEFs e residentes foram convidados a participar das intervenções e orientá-las juntamente com a equipe de saúde.

As variáveis coletadas e analisadas a partir dos prontuários foram: idade, motivo de internação (TP, diabetes gestacional, síndromes hipertensivas e outros), tipo de via de parto (vaginal, vaginal com uso de fórceps e cesáreo), métodos não farmacológicos para o alívio da dor aplicados às parturientes em SPPH por PEF. Estes dados foram tabulados em planilha própria de coleta Microsoft Office Excel para posterior análise. Foi realizada análise descritiva mediante o software Stata, versão 14, para avaliar as frequências relativas e absolutas das variáveis mencionadas.

No que tange as entrevistas (GAUTHIER, 1998), elas foram individuais, com os profissionais de saúde atuantes na SPPH (enfermeiros, técnicos em enfermagem e obstetras), exceto aqueles $\mathrm{PEF}$; realizadas por um profissional da área da odontologia, não participante do espaço estudado, e conhecedor de metodologia qualitativa. Todos os profissionais efetivos que atuavam na SPPH a pelo menos seis meses (SOUZA; GAÍVA; MODES, 2011) e que trabalharam neste cenário na presença de PEF foram convidados a fazer parte do estudo.

O convite ocorreu mediante contato com a chefia do setor, disponibilidade da lista de funcionários atuantes no setor a mais de seis meses, e posterior contato do profissional entrevistador com os funcionários, de forma aleatória, averiguando disponibilidade para participar do estudo e haver atuado com PEF em SPPH. De posse dessas informações, se agendava a entrevista, realizada em ambiente privado, em área de orientação acadêmica do Hospital Escola, uma vez assinado o termo de consentimento livre e esclarecido. Para nominar os participantes, utilizaram-se siglas, seguidas por um numeral, por exemplo: Profissional 1 (P1) e assim por diante, garantindo sua confidencialidade e privacidade de acordo com a Resolução nº. 466/2012 (BRASIL, 2012).

Ao longo de seis semanas, um profissional da área da odontologia, não participante do espaço estudado, e conhecedor de metodologia qualitativa, realizou as entrevistas que foram gravadas por dispositivo de áudio, sendo efetuadas no máximo três em um mesmo dia da semana. O número de participantes foi definido pelo critério de saturação dos dados, no qual o pesquisador interrompe as entrevistas quando após as informações coletadas com um determinado número de pessoas, novas entrevistas passam a apresentar uma quantidade de repetições em seu conteúdo (TURATO, 2003).

Para análise das entrevistas, inicialmente foram transcritas pela primeira autora deste artigo, e revisadas pelos entrevistados, para posterior análise de conteúdo preconizada pela técnica de Bardin, a qual se constitui no conjunto de técnicas de análise das comunicações que utiliza procedimentos sistemáticos e objetivos de descrição do conteúdo das mensagens. Essa técnica é composta por três etapas, sendo a primeira definida como pré-análise, que consiste na organização do material a ser analisado com o objetivo de sistematizar as ideias iniciais. A segunda etapa baseia-se pela exploração do material, que consiste na definição de categorias e identificação das unidades de registro e de contexto das falas das participantes. Daqui, foram extraídas duas categorias de análise, a primeira sendo "O PEF como integrante da equipe multiprofissional" e a segunda sendo "O PEF no cuidado a parturiente" a partir das seguintes perguntas norteadoras da pesquisa: "Sobre a composição da equipe de saúde que atende a SPPH você entende que o PEF pode contribuir neste espaço?"; "Você entende que o PEF oferece apoio empático às mães da SPPH?" e, "você entende que o PEF auxilia na redução de dor, ou na melhor forma de manejar a mesma?". Por fim, foi realizada a terceira etapa que se baseia no tratamento e interpretação dos resultados, com destaque das informações para análise, culminando nas interpretações inferenciais (BARDIN, 2010).

\section{RESULTADOS E DISCUSSÃO}

Observamos que, no período de março de 2016 a agosto de 2017, os PEF atuaram em 59 atendimentos realizados pela equipe multiprofissional em SPPH com o uso de métodos não farmacológicos para alívio da dor com as parturientes e prestando os auxílios demandados pela equipe de enfermagem. As gestantes atendidas tinham, em sua maioria, idades entre 21 e 30 anos de idade $(47,5 \%)$, tendo sido internadas prioritariamente por TP (61\%), resultando predominantemente em partos realizados por via vaginal $(74,6 \%)$ (Tabela 1).

Tabela 1. Idade, motivo de internação e desfecho de parto das pacientes atendidas por equipe multiprofissional em SPPH na presença de PEF $(n=59)$.

\begin{tabular}{|c|c|c|c|c|c|c|}
\hline \multirow{2}{*}{ Variáveis } & \multicolumn{2}{|c|}{ Geral } & \multicolumn{2}{|c|}{2016} & \multicolumn{2}{|c|}{2017} \\
\hline & $\mathrm{n}$ & $\%$ & $\mathrm{n}$ & $\%$ & $\mathrm{n}$ & $\%$ \\
\hline \multicolumn{7}{|l|}{ Idade (anos) } \\
\hline$<21$ & 19 & 32,2 & 10 & 31,2 & 9 & 33,3 \\
\hline 21 a 30 & 28 & 47,5 & 15 & 46,9 & 13 & 48,2 \\
\hline$>30$ & 12 & 20,3 & 7 & 21,9 & 5 & 18,5 \\
\hline \multicolumn{7}{|l|}{ Motivo da Internação } \\
\hline Trabalho de Parto & 36 & 61 & 17 & 53,1 & 19 & 70,4 \\
\hline Diabetes Gestacional & 1 & 1,7 & 1 & 3,1 & & \\
\hline Síndromes Hipertensivas & 5 & 8,5 & 2 & 6,3 & 3 & 11,1 \\
\hline Outros & 17 & 28,8 & 12 & 37,5 & 5 & 18,5 \\
\hline \multicolumn{7}{|l|}{ Via de Parto } \\
\hline Vaginal & 44 & 74,6 & 22 & 68,8 & 22 & 81,4 \\
\hline Vaginal com Fórceps & 3 & 5,1 & 1 & 3,1 & 2 & 7,4 \\
\hline Cesáreo & 12 & 20,3 & 9 & 28,1 & 3 & 11,1 \\
\hline
\end{tabular}

Referente aos métodos não farmacológicos para alívio de dor (Tabela 2) utilizados por PEF nos atendimentos às parturientes verificados o uso das seguintes técnicas: movimentação pélvica em bola suíça, caminhadas, banho de aspersão, modificações de postura e massagens - principalmente na região lombar. Apenas $45,8 \%$ das intervenções utilizam apenas uma das técnicas de forma isolada, sendo todas as demais intervenções com a associação de mais de uma das técnicas relatadas. A técnica mais fre- 
quentemente utilizada de forma isolada foi a movimentação em bola suíça (22\%) seguida das caminhadas (13,6\%). Estas técnicas também são aquelas mais utilizadas de forma combinada $(18,6 \%)$ seguida pela combinação da movimentação em bola suíça com o banho de aspersão (11,9\%).

Tabela 2. Métodos não farmacológicos para alívio de dor realizado por PEF de forma isolada ou combinada $(n=59)$.

\begin{tabular}{|c|c|c|c|c|c|c|}
\hline \multirow{2}{*}{ Variáveis } & \multicolumn{2}{|c|}{ Geral } & \multicolumn{2}{|c|}{2016} & \multicolumn{2}{|c|}{2017} \\
\hline & $\mathrm{n}$ & $\%$ & $\mathrm{n}$ & $\%$ & $\mathrm{n}$ & $\%$ \\
\hline \multicolumn{7}{|l|}{ Intervenções isoladas } \\
\hline Bola suíça & 13 & 22 & 6 & 18,7 & 7 & 26 \\
\hline Banho de aspersão & 4 & 6,8 & 1 & 3,1 & 3 & 11,1 \\
\hline Caminhadas & 8 & 13,6 & 5 & 15,6 & 3 & 11,1 \\
\hline Massagens & 1 & 1,7 & - & - & 1 & 3,7 \\
\hline Modificações de postura & 1 & 1,7 & - & - & 1 & 3,7 \\
\hline \multicolumn{7}{|l|}{$\begin{array}{l}\text { Intervenções combinadas com } \\
\text { duas técnicas }\end{array}$} \\
\hline $\begin{array}{l}\text { Bola suíça e Banho de } \\
\text { aspersão }\end{array}$ & 7 & 11,9 & 3 & 9,4 & 4 & 14,8 \\
\hline Bola suíça e Caminhadas & 11 & 18,6 & 9 & 28,1 & 2 & 7,4 \\
\hline Caminhadas e Massagens & 3 & 5,1 & 3 & 9,3 & - & - \\
\hline $\begin{array}{l}\text { Caminhadas e Banho de } \\
\text { aspersão }\end{array}$ & 3 & 5,1 & 1 & 3,1 & 2 & 7,4 \\
\hline \multicolumn{7}{|l|}{$\begin{array}{l}\text { Intervenções combinadas } \\
\text { com três técnicas }\end{array}$} \\
\hline $\begin{array}{l}\text { Caminhadas, Bola suíça e } \\
\text { Banho de aspersão }\end{array}$ & 4 & 6,8 & 2 & 6,2 & 2 & 7,4 \\
\hline $\begin{array}{l}\text { Caminhadas, Bola suíça e } \\
\text { Massagens }\end{array}$ & 1 & 1,7 & 1 & 3,1 & - & - \\
\hline $\begin{array}{l}\text { Caminhadas, Banho de } \\
\text { aspersão e Massagens }\end{array}$ & 2 & 3,4 & 1 & 3,1 & 1 & 3,7 \\
\hline $\begin{array}{l}\text { Bola suíça, Banho de aspersão } \\
\text { e Massagens }\end{array}$ & 1 & 1,7 & - & - & 1 & 3,7 \\
\hline
\end{tabular}

Conforme critério de saturação dos dados, descrito na metodologia, oito foram os profissionais de saúde entrevistados, sendo quatro enfermeiros e quatro técnicos de enfermagem. Destes, seis possuíam nível superior de ensino e dois, formação de nível técnico. Dos seis profissionais com nível superior, quatro possuíam especialização e um doutorado.

Ao analisar a percepção desses profissionais de saúde atuantes na SPPH, referente a contribuição do PEF em SPPH por meio da pergunta norteadora "Sobre a composição da equipe de saúde que atende a SPPH, você entende que o PEF pode contribuir neste espaço?" observa-se pela análise das transcrições, que o PEF é entendido como um profissional que complementa e qualifica a equipe multiprofissional, conforme falas que se seguem:

"Ah, eu acredito que ele nos auxilia nessa parte de condução. É importante pra qualificar o atendimento da equipe toda" (P4)

"Acho que é um complemento para equipe, pra dar uma qualidade de atendimento pra gestante" (P1)

“[...] pra ajudar as mães a fazer os exercícios né, porque a gente sabe o básico, só o básico mesmo né; porque a gente bota na bola" (P2)

"Acho que ajuda bastante a gestante nesse momento, no alívio da dor, no cuidado dela em geral, junto com a equipe" (P6)

Ah, até por causa das mães né, elas se sentem mais relaxadas [...] não é todos os hospitais que têm um PEF até pra elas, segurança para elas é tudo" (P2)

"Acho que esta atuação do PEF junto com a enfermagem no TP das gestantes seja menos traumático do que a gente vê" (P7)

Entretanto, também podemos observar que as respostas são amplas, o que pode dever-se a diferentes fatores como, por exemplo, o desconhecimento do que seria o papel do PEF no setor, respondendo a partir da observação dos comportamentos das parturientes. Nas falas de P2 observamos uma percepção de aporte dos PEF quanto a conhecimentos relacionados a exercícios e um reflexo positivo quanto à confiança e sensação de menor tensão das parturientes. Com a contribuição de P6 percebemos um resultado efetivo de alívio de dor. Por outro lado, na fala de P7 podemos constatar uma possível resistência inicial a presença do PEF no cenário, que aparentemente percebe-se sendo desfeita com as atuações multiprofissionais. Entretanto, se por um lado sabemos da importância do atendimento multiprofissional para a saúde, por outro, o preconizado pela Organização Mundial da Saúde é o atendimento interprofissional, que exige além de atuações sobrepostas atuações efetivamente em conjunto (ROSSIT et al., 2018). Desafio que está estabelecido há alguns anos e que ainda carece de atenção (PEDUZZI, 2016).

Em relação a segunda questão sobre o apoio às gestantes: "Você entende que o PEF oferece apoio empáticos às mães da SPPH?", observa-se que alguns dos comentários mostraram que os profissionais de saúde acreditam que as gestantes percebem o PEF como um profissional relacionado ao bem-estar e, portanto, aceitam as propostas dos métodos não farmacológicos para alívio da dor de melhor grado, conforme podemos observar nas seguintes transcrições que enaltecem as ideias pré-concebidas de cada área profissional:

\footnotetext{
“...é muito mais fácil quando tem o PEF porque a gestante nos vê de uma forma e é claro a gente atua, a gente ajuda na bola, a gente coloca no chuveiro, mas quando entra o PEF eles já se identificam oi, boa tarde, bom dia, eu sou o PEF aqui do hospital, vamos fazer os exercícios, aí eles ligam uma imagem a outra aí eles não resistem mais" (P5)

"Com a gente da enfermagem eles nos veem com mais dor parece, acho que tem aquela coisa, ah vão me furar, ah vão me fazer a medicação, eles veem dessa forma a gente" (P3)
}

Por fim, quando perguntados se o PEF auxilia no tratamento da dor, pelo seguinte questionamento: "Você entende que o PEF auxilia na redução de dor, ou na melhor forma de afrontar a mesma?" As falas dos profissionais de saúde demonstraram que a atuação do PEF é percebida de forma positiva ainda que nem todas expressem as mesmas ideias:

[...] de confrontar a dor sim, porque tem a respiração tudo e tal, ajuda mas aquilo dali não tem o que tu faça é TP" (P2)

[...] ah, tem a questão do banho né, que as gurias da educação física trabalham muito com a questão do banho e ajuda bastante na dor, porque elas chegam com um grau de dor assim 8 e elas vão pro banho e daí fica 6, 4 e baixa bastante" (P3) 
"Sim, nesse sentido mesmo de ficar auxiliando na parturiente nas questões não farmacológicas da dor" (P8)

"De evolução do parto e amenizar a dor e eu acho que nessas funções é muito importante sim" (P6)

Na fala de P2 observamos que apesar de acreditar que sim auxilia no alívio da dor, esse alívio não chega a ser significativo, dado que é um processo extremamente doloroso. E, além dos exercícios descritos, destaca a importância da respiração na execução das atividades. Prática esta que está relacionada à diminuição da ansiedade e a técnicas de relaxamento (BÕING; SPERANDIO; SANTOS, 2007). A fala de $\mathrm{P} 3$ retira qualquer dúvida quanto ao efeito do trabalho do PEF, dado que existe uma medida que aponta a efetiva redução. Claro que, novos estudos são necessários para avaliar o quão significativo são esses resultados e se realmente se deve a atuação do PEF. As demais falas efetivamente corroboram com o que os demais profissionais de saúde percebem e entendem do papel do PEF em SPPH, e sim, reconhecem sua atuação vinculada ao alívio da dor de forma não farmacológica, coincidindo com a descrição da literatura a respeito do uso das técnicas utilizadas pelos PEF (CAVALCANTI et al., 2019; APOLINÁRIO et al., 2016).

Este estudo buscou descrever as intervenções realizadas, em SPPH, por PEF junto às parturientes, e a percepção dos demais profissionais de saúde sobre sua atuação no atendimento em equipe multiprofissional neste cenário. Neste sentido, observou-se que das técnicas mais utilizadas pelos PEF para o alívio da dor por método não farmacológico, estão: movimentação pélvica com o uso de bola suíça, caminhada e banho de aspersão, utilizadas com maior frequência de forma combinadas. E, que o PEF é percebido pelos demais profissionais de saúde que atuam na SPPH como um profissional que pode auxiliar a equipe, promover alívio de dor e melhorar o bem-estar das parturientes.

No que se refere ao uso das técnicas não farmacológicas para o alívio de dor, é conhecido que tanto o uso delas de forma isolada ou combinada demonstra alívio da dor e aumento da sensação de bem-estar durante o TP (CAVALCANTI et al., 2019). A literatura tem demonstrado aplicação mais frequente das técnicas de forma combinada conforme tempo de TP e autonomia da parturiente (APOLINÁRIO et al., 2016). Inclusive, estudo relata que as estratégias não farmacológicas combinadas na fase ativa do TP diminuíram a percepção de dor das mulheres após a realização dos métodos aos seis, oito e nove centímetros de dilatação cervical, resultado contrário ao esperado em tal condição fisiológica (DAVIM; TORRES; MELO, 2007).

Técnicas previamente relatadas na literatura como sendo mais utilizadas - movimentação pélvica com o uso da bola suíça e as caminhadas - foram aquelas aplicadas com maior frequência nos atendimentos, tanto de forma isolada como combinada. O uso da bola suíça é um importante recurso não medicamentoso para alívio da dor da paciente (OLIVEIRA; CRUZ, 2014) devido ao balanceio pélvico permitindo atuação direta na musculatura pélvica, relaxando e alongando principalmente nos músculos levantadores do ânus, pubo coccígeos e a fáscia da pelve (SILVA et al., 2011).

Assim, a adoção de posturas verticais associadas à movimentação pode minimizar a dor sentida pela parturien- te além de aumentar a eficácia das contrações uterinas, melhorando a circulação sanguínea materno-fetal e minimizando a ocorrência do trauma perineal (PAIVA, 2018). Além disso, o estímulo à mobilidade materna durante o processo de parturição produz efeitos benéficos como maior resistência a dor devido a mudança de diâmetro da pelve na posição vertical, que favorece a intensidade das contrações uterinas e o ajuste mais rápido da cabeça fetal na bacia materna (GALO et al., 2014).

O banho de aspersão, que também apareceu como uma das técnicas bastante utilizada, principalmente de forma combinada e, de acordo com a literatura, está associada à capacidade de relaxamento e consequente sensação de alívio das algias (RITTER, 2012); pelo que parece que deve ser estimulada segundo os dados da literatura e aqueles corroborados em fala de profissional de enfermagem deste estudo (SILVA et al., 2011).

Entende-se que as técnicas não farmacológicas utilizadas para alívio de dor estão associados com procedimentos que os PEF dominam, e isso, de certa forma, também apareceu na descrição de maior aceitação por parte das parturientes ao convite dos PEF à participação nas técnicas de alívio de dor não farmacológicas descrita por profissional da equipe de enfermagem. Também está descrito na literatura que exercícios físicos contribuem para alívio de dor (ABREU et al., 2018) e que a utilização de movimentação com o uso de bola suíça, mediante caminhadas, relacionadas a manutenção da força muscular e da flexibilidade principalmente da mobilidade pélvica, tem demonstrado trazer benefícios a gestantes tanto de forma aguda como crônica (SOARES et al., 2017; CONNOLLY et al., 2019; LEUNG et al., 2013), da mesma forma, atividades como massagens e o uso de calor com banhos e bolsas térmicas são conhecidas do PEF na atuação junto à atletas, por exemplo, para alívio de dor e desconforto pós atividades esportivas (CELI NETO, 2020). Assim, ressalta-se a importância dos resultados deste estudo e que o PEF pode manter suas potencialidades de atuação profissional também em SPPH.

Estudos contemplando a percepção de atuação do PEF na literatura, conforme previamente comentado, ainda são escassos, o que enaltece a relevância desta pesquisa. Conforme os relatos da equipe de enfermagem (enfermeiros e técnicos de enfermagem), o PEF contribui com a parturiente e contribui com a equipe, o que por si só já é um resultado relevante e que condiz com o descrito até aqui. Resultados positivos para a atuação de PEF como membro de equipes multiprofissionais em áreas de reabilitação cardíaca e tratamento de doenças crônicas são verificados e estão estabelecidos, dentre outros fatores, devido a sua ampla visão em saúde do ponto de vista terapêutico e preventivo (VIEIRA, 2013). O fato de perceber o PEF como àquele ligado à sensação de bem-estar também já foi descrita na literatura em estudo realizado na atenção básica (SAPORETTI; MIRANDA; BELISÁRIO, 2016), o que fortalece a criação de vínculos e a maior adesão à intervenções/tratamentos. Por outro lado, sabemos que um profissional não faz saúde e que todos os profissionais de saúde são necessários para a saúde das pessoas e da população e que uma maior interação entre todos deve ser estimulada.

Ressalta-se, portanto, que PEF tem a contribuir em SPPH em conjunto com a equipe multiprofissional que ali atua. O alívio da dor mediante diferentes técnicas foi o 
destacado na participação desse profissional pela equipe. Entretanto, outras pesquisas são necessárias envolvendo um maior número de profissionais, levando em conta outras condições clínicas das gestantes, ampliando o olhar da atuação do PEF em equipe multiprofissional em atendimentos em SPPH, sobre a efetividade das técnicas nas percepções das parturientes, bem como, sobre fase ativa da parturição, trauma perineal, evolução de parto para via vaginal, entre outros.

Alguns aspectos carecem de ser considerados para a análise dos resultados apresentados nesse estudo. O primeiro deles é que a amostra é pequena e se refere a um contexto específico, sendo necessários novos estudos sobre o tema. Outro, é referente a que os atendimentos realizados na presença de PEF, em sua maioria, foram a parturientes em faixa considerada ideal para gestação, o que permite uma intervenção mais efetiva do PEF e aumenta a probabilidade de melhores desfechos em saúde tanto maternos quanto perinatais (SCHUPP et al., 2006). Da mesma forma, ao predominar como motivo de internação o TP, podemos deduzir que essas gestantes apresentavam menor probabilidade de disporem de complicações relacionadas à gestação. Informações essas que devem ser consideradas para futuros estudos.

\section{CONCLUSÃO}

Ao buscar compreender as diferentes interfaces de atuação do profissional de Educação Física em SPPH com outros profissionais, observamos que tanto na literatura quando nos relatos aqui trazidos, as possibilidades de avanços nas intervenções favorecem uma melhor atenção às parturientes.

Constatou-se que os atendimentos dos PEF em SPPH em um hospital universitário do sul do Brasil estão centrados em intervenções combinadas fundamentalmente com movimentação pélvica com o uso de bola suíça e caminhadas, sendo conhecidas e aplicadas por PEF, dando a este um novo espaço de reconhecimento profissional.

Também se encontrou uma percepção positiva dos profissionais de saúde que atuam na SPPH, reconhecendo a importância do PEF as e suas competências de atuação para trazer melhores benefícios as pacientes e à equipe. Cabe ainda destacar que outros estudos são necessários nesta perspectiva que avaliem a percepção das parturientes frentes aos atendimentos por equipes multiprofissionais objetivando aprimorar a efetividade dos métodos não farmacológicos para alívio da dor em diferentes desfechos relacionados ao TP.

\section{AGRADECIMENTOS}

Os autores agradecem a equipe de sala de pré-parto do Hospital Escola da Universidade Federal de Pelotas, Rio Grande do Sul, Brasil.

\section{CONFLITO DE INTERESSE}

Os autores do estudo declaram não haver conflito de interesses.

\section{FINANCIAMENTO}

Este estudo não teve apoio financeiro.

\section{REFERÊNCIAS}

ABREU, W. C.; FAGUNDES, F. R. C.; MIYAMOTO, G. C.; CABRAL, C. M. N. Revisão sistemática sobre exercício no tratamento da dor lombar e pélvica gestacional. ConScientiae Saúde, São Paulo, v. 17, n. 3, p. 355-41, 2018. Disponível em: https://periodicos.uninove.br/saude/article/view/8508/5067.

ALVES, T. V.; BEZERRA, M. M. M. Principais alterações fisiológicas e psicológicas durante o Período Gestacional. Id on Line Revista de Psicologia, Piedade, v. 14, n. 49, p. 114-26, 2020. DOI: https://doi.org/10.14295/idonline. v14i49.2324.

APOLINÁRIO, D: RABELO, M. WOLFF, L. D. G.: SOUZA, S. R. R. K: LEAL, G. C. G. Práticas na atenção ao parto e nascimento sob a perspectiva das puérperas. Revista Rene, Fortaleza, v. 17, n. 1, p. 20-8, 2016. DOI: https://doi. org/10.15253/2175-6783.2016000100004.

BARDIN, L. Análise de conteúdo. Lisboa: Edições 70, 2010.

BÕING, I.; SPERANDIO, F. F.; SANTOS, G. M. Uso de técnica respiratória para analgesia no parto. Femina, São Paulo, v. 35, n. 1, p. 41-6, 2007.

BRASIL. Ministério da Saúde. Secretaria de Ciência, Tecnologia e Insumos Estratégicos. Departamento de Gestão e Incorporação de Tecnologias em Saúde. Diretrizes nacionais de assistência ao parto normal: versão resumida. Disponível em: <https://bvsms.saude.gov.br/bvs/publicacoes/diretrizes nacionais assistencia parto normal.pdf> Acessado em: 24 de janeiro de 2020.

BRASIL. Portaria $n^{\circ}$ 687, de 30 de março de 2006. Aprova a Política de Promoção da Saúde. Série Pactos pela Saúde 2006. Brasília, DF, v. 7. 2010. Disponível em: <https://bvsms.saude.gov.br/bvs/saudelegis/gm/2006/ prt0687 3003 2006.html>. Acessado em: 02 de janeiro de 2016.

BRASIL. Portaria n 154, de 24 de janeiro de 2008. Cria os Núcleos de Apoio à Saúde da Família - NASF. Brasília, DF. 2008. Disponível em: <https://bvsms.saude.gov.br/bvs/saudelegis/gm/2008/prt0154_24_01_2008.html>. Acessado em: 02 de janeiro de 2016.

BRASIL. Portaria $n^{\circ}$ 3.390, de 30 de dezembro de 2013. Institui a Política Nacional de Atenção Hospitalar (PNHOSP) no âmbito do Sistema Único de Saúde (SUS), estabelecendo- se as diretrizes para a organização do componente hospitalar da Rede de Atenção à Saúde (RAS). Brasília, DF. 2013. Disponível em: <https://bvsms.saude.gov.br/bvs/saudelegis/gm/2013/ prt3390 3012 2013.html>. Acessado em: 02 de janeiro de 2016.

BRASIL. Portaria $n^{\circ} 569$, de $1^{\circ}$ de junho de 2000 . Instituir o Programa de Humanização no Pré-natal e Nascimento, no âmbito do Sistema Único de Saúde 2000. Disponível em: <https://bvsms.saude.gov.br/bvs/saudelegis/ gm/2000/prt0569 01062000 rep.html>. Acessado em: 02 de janeiro de 2016.

BRASIL. Resolução n. ${ }^{\circ}$ 287, de 08 de outubro de 1998. Relaciona categorias profissionais de saúde de nível superior para fins de atuação do CNS. Diário Oficial União, Brasília, DF. 15 de julho de 2003. Seção1. Disponível em: <https://bvsms.saude.gov.br/bvs/saudelegis/cns/1998/res0287 08 10_1998. html>. Acessado em: 02 de janeiro de 2016

BRASIL. Resolução n 466, de 12 de dezembro de 2012. Aprovar as seguintes diretrizes e normas regulamentadoras de pesquisas envolvendo seres humanos. 2012. Disponível: <https://bvsms.saude.gov.br/bvs/saudelegis/ cns/2013/res0466 12 12 2012.html> Acessado em: 24 de janeiro de 2020.

CARVALHO, F. F. B. Práticas corporais e atividades físicas na atenção básica do sistema único de saúde: ir além da prevenção das doenças crônicas não transmissíveis é necessário. Movimento, Porto Alegre, v. 22, n. 2, p. 647-58, 2016. DOI: https://doi.org/10.22456/1982-8918.58174.

CAVALCANTI, A. C. V.; HENRIQUE, A. J.; BRASIL, C. M.; GABRIELLONI, M. C.; BARBIERI, M. Terapias complementares no trabalho de parto: ensaio clínico randomizado. Revista Gaúcha de Enfermagem, Porto Alegre, v. 40, p. 26, 2019. DOI: https://doi.org/10.1590/1983-1447.2019.20190026.

CELI NETO, ।.: MATOS, M. D. S.; PINHEIRO, R. D. V.; NERI, S. G. R.; MELO, L. M. A. B. D.; RIBEIRO, A. L. D. A. Efeitos da massagem rápida no estresse de praticantes de atividade física. Lecturas Educación Física y Deportes, Revista Digital, Buenos Aires, v. 24, n. 262, p. 73-57, 2020. Disponível em: https://www.efdeportes.com/efdeportes/index.php/EFDeportes/article/ view/508/1146.

COELHO, C. F.; BURINI, R. C. Atividade física para prevenção e tratamento das doenças crônicas não transmissíveis e da incapacidade funcional. Revista de Nutrição de Campinas, Campinas, v. 22, n. 6, p. 937-46, 2009. DOI: https://doi.org/10.1590/S1415-52732009000600015.

CONNOLLY, C. P.; CONGER, S. A.; MONTOYE, A. H. K.; MARSHALL, M. R.; SCHLAFF, R. A.; BADON, S. E.; PIVARNIK, J. M. Walking for health during pregnancy: A literature review and considerations for future research. Journal of Sport and Health Science, Shanghai, v. 8, n. 5, p. 401-11, 2019. DOI: https:// doi.org/10.1016/j.jshs.2018.11.004. 
DAVIM, R. M. B.; TORRES, G. V.; MELO, E. S. Estratégias não farmacológicas no alívio da dor durante o trabalho de parto: pré-teste de um instrumento. Revista Latino-americana de Enfermagem, Ribeirão Preto, v. 15, n. 6, p. 1156-60, 2007. DOI: https://doi.org/10.1590/S0104-11692007000600015.

EBSERH. Empresa Brasileira de Serviços Hospitalares. Apresentação - EBSERH. Disponível em: <http://portal.mec.gov.br/ebserh--empresa-brasileira-de-servicos-hospitalares\#: :text=A\%20 Empresa\%20Brasileira\%20de\%20Servi\%C3\%A7os,apoio\%20diagn\%C3\%B3stico\%20e\%20terap\%C3\%AAutico\%20\%C3\%A0>. Acessado em: 24 de janeiro de 2020

FERREIRA, J. C. V.; FERREIRA, J. S. Atuação dos profissionais de educação física na atenção primária à saúde. Caderno de Educação Física e Esporte, Marechal Cândido Rondon, v. 15, n. 2, p. 113-5, 2017. DOI: https://doi. org/10.36453/2318-5104.2017.v15.n2.p105.

GALLO, R. B.; SANTANA, L. S.; MARCOLIN, A. C.; QUINTANA, S. M. A bola suíça no alívio da dor de primigestas na fase ativa do trabalho de parto. Revista Dor, São Paulo, v. 15, n. 4, p. 253-5, 2014. DOI: https://doi.org/10.5935/18060013.20140054.

GAUTHIER, J. H. M. Pesquisas em enfermagem novas metodologias aplicadas. Rio de Janeiro: Guanabara Koogan; 1998.

LEUNG, R. W.; LI, J. F.; LEUNG, M. K.; FUNG, B. K.; FUNG, L. C.; TAI, S. M.; SING, C.; LEUNG, W. C. Efficacy of birth ball exercises on labour pain management. Hong Kong Medical Journal, Hong Kong, v. 19, n. 5, p. 393-9, 2013. DOI: https://doi.org/10.12809/hkmj133921.

LIMA, F. J. B.: SOUSA, N. M.: PINTO, A. C. M. D. Relação do tipo de parto na constituição da microbiota infantil. In: Encontro de Extensão e Docência e Iniciação Científica (EEDIC). Anais... Local: Editora: 2019. Disponível em: $<$ http://publicacoesacademicas.unicatolicaquixada.edu.br/index.php/eedic/article/view/3144/2692>, Acessado em: 23 de março de 2020.

LOGAN, J. G. Effects of stretching exercise on heart rate variability during pregnancy. Journal of Cardiovascular Nursing, Philadelphia, v. 3, n. 2, p. 107-11, 2016. DOI: https://doi.org/10.1097/JCN.0000000000000326.

MAFETONI, R. R.; SHIMO, A. K. K. Métodos não farmacológicos para alívio da dor no trabalho de parto: revisão integrativa. Revista Mineira de Enfermagem, Belo Horizonte, v. 18, n. 2, p. 512-5, 2014. DOI: https://doi. org/10.5935/1415-2762.20140037.

MELO, R. C. S.; DALLA DÉA, V. H. S. Atuação do professor de educação física em uma maternidade de Goiânia/GO. In: XVIII Congresso Brasileiro de Ciências do Esporte e $V$ Congresso Internacional de Ciências do Esporte. Anais... Brasília, DF: CBCE, 2013. Disponível em: <https://old.cev.org.br/arquivo/biblioteca/4014300.pdf>. Acessado em: 20 de março de 2018.

OLIVEIRA, L. M. N.; CRUZ, A. G. C. A Utilização da bola suíca na promoção do parto humanizado. Revista Brasileira de Ciências da Saúde, João Pessoa, v. 18, n. 2, p. 175-80, 2014. DOI: https://doi.org/10.4034/RBCS.2014.18.02.13.

OMS. Organização Mundial da Saúde. Diretrizes da OMS para atividade física e comportamento sedentário. Disponível: <https://apps.who.int/ iris/bitstream/handle/10665/337001/9789240014886-por.pdf?sequence $=102 \&$ isAllowed $=y>$. Acessado em: 22 de janeiro de 2020 .

PAIVA, E. F.; STHAL, H. C.; PAULINO, V. C. P.; LEITE, G. R. Posições assumidas durante o parto normal: percepção de puérperas atendidas numa maternidade de Jataí - Goiás. Itinerarius Reflectionis, Jataí, v. 14, n. 4, p. 1-21, 2018. DOI: https://doi.org/10.5216/rir.v14i4.54977.

PEDUZZI, M. O SUS é interprofissional. Revista Interface, Botucatu, v. 20 n. 56, p. 201-199, 2016. DOI: https://doi.org/10.1590/1807-57622015.0383.

PIMENTEL, T. A.; OLIVEIRA FILHO, E. C. Fatores que influenciam na escolha da via de parto cirúrgica: uma revisão bibliográfica. Universitas: Ciências da Saúde, Brasília, v. 14, n. 2, p. 199-87, 2016. DOI: https://doi.org/10.5102/ ucs.v14i2.4186.

RAMIRES, V. V. BECKER, L. A.; SADOVSKY, A. D. I.: ZAGO, A. M.; BIELEMANN, R. M. GUERRA, P. H. Evolução da pesquisa epidemiológica em atividade física e comportamento sedentário no Brasil: atualização de uma revisão sistemática. Revista Brasileira de Atividade Física e Saúde, Florianópolis, v. 19, n. 5, p. 529-47, 2014. DOI: https://doi.org/10.12820/rbafs.v.19n5p529.

RITTER, K. M. Manejo não farmacológico da dor em mulheres durante o trabalho de parto em um hospital escola. 2012.77f. Dissertação (Mestrado em Enfermagem) - Universidade Federal do Rio Grande do Sul, Porto Alegre, 2012. Disponível em: <https://lume.ufrgs.br/handle/10183/69750\#: :tex$\mathrm{t}=$ Os $\% 20 \mathrm{~m} \%$ C3\%A9todos\%20n\%C3\%A30\%20farmacol\%C3\%B3gicos\%20 (MNF,MNF\%20de\%20al\%C3\%ADvio\%20da\%20dor>,

ROSSIT, R. A. S. FREITAS, M. A. O. BATISTA, S. S. H. S. BATISTA, N. A. Constructing professional identity in interprofessional health education as perceived by graduates. Revista Interface, Botucatu, v. 22, p. 1399-410, 2018. DOI: https://doi.org/10.1590/1807-57622017.0184.

SAPORETTI, G. M.; MIRANDA, P. S. C.; BELISÁRIO, A. S. O profissional de edu- cação física e a promoção da saúde em núcleos de apoio à saúde da família. Revista Trabalho, Educação e Saúde, Manguinhos, v. 14, n. 2, p. 523-43, 2016. DOI: https://doi.org/10.1590/1981-7746-sip00113.

SCABAR, T. G.; PELICIONI, A. F.; PELICIONI, M. C. F. Atuação do profissional de educação física no sistema único de saúde: uma análise a partir da Política Nacional de Promoção da Saúde e das Diretrizes do Núcleo de Apoio à Saúde da Família - NASF. Journal of the Health Sciences, São Paulo, v. 30 , n. 4, p. 411-18, 2012. Disponível em: <https://repositorio.unip.br/journal-of-the-health-sciences-institute-revista-do-instituto-de-ciencias-da-saude/>.

SCHUPP, T. R. Gravidez após os 40 anos de idade: análise dos fatores prognósticos para resultados maternos e perinatais diversos. 2006. $162 \mathrm{f}$. Tese (Doutorado em Ginecologia e Obstetrícia) - Universidade de São Paulo São Paulo, 2006. Disponível em: <https://www.teses.usp.br/teses/disponiveis/5/5139/tde-03052007-142303/pt-br.php>.

SILVA, L. M.; OLIVEIRA, S. M.; SILVA, F. M., Alvarenga, M. B. Uso da bola suíça no trabalho de parto. Acta Paulista de Enfermagem, São Paulo, v. 24, n. 5, p. 656-62, 2011. DOI: https://doi.org/10.1590/S0103-21002011000500010.

SOARES, D. S. C.; SOARES, J. J.; GRAUP, S.; STREB, A.R. Atividade física na gestação: uma revisão integrativa. Revista Perspectiva: Ciência e Saúde, Osório, v. 2, n. 2, p. 84-71, 2017. DOl: https://doi.org/10.1590/S010321002011000500010 .

SOUZA, T. G.; GAÍVA, M. A. M.; MODES, P. S. S. A. A humanização do nascimento: percepção dos profissionais de saúde que atuam na atenção ao parto. Revista Gaúcha de Enfermagem, Porto Alegre, v. 32, n. 3, p. 47986, 2011. Disponível em: <https://1library.org/document/oy81x62q-humanizacao-nascimento-percepcao-profissionais-saude-atuam-atencao-parto. html>.

SPERLING, S. G.; ROMAN, A. R.; GOMES, J. S.; PORTELLA, M. P.; KIRCHNER, R. M.: STUMM, E. M. Dor e estresse percebido em mulheres no pós-parto vaginal. Revista Dor, São Paulo, v. 7, n. 4, p. 289-93, 2016. DOI: https://doi. org/10.5935/1806-0013.20160091.

STEINHILBER, J. Resolução $n^{\circ}$ 391/2020. Reconhece que o Profissional de Educação Física. Conselho Federal de Educação Física. Disponível em: $<$ https://www.in.gov.br/web/dou/-/resolucao-n-391-de-26-de-agosto-de-2020-274726255>. Acessado em: 22 de janeiro de 2020.

TURATO, E. R. Tratado da metodologia da pesquisa clínico-qualitativa. Petrópolis: Editora Vozes, 2003.

VIEIRA, M. C. A atuação do professor de educação física na reabilitação cardíaca: possibilidades e desafios. Arquivos em Movimento, Rio de Janeiro, v. 9, n. 2, p. 102-8, 2013. Disponível em: <https://revistas.ufri.br/index.php/ am/article/view/9226/pdf 25>.

\section{ORCID E E-MAIL DOS AUTORES}

Juliana Quadros Santos Rocha (Autor Correspondente)
(iD https://orcid.org/0000-0002-9743-6331

@ julianaqrocha2@gmail.com

Lidiane Pozza Costa

(iD http://orcid.org/0000-0003-4551-5465

Q lidiane.pozza@ebserh.gov.br

Marcelo Zanusso Costa

(iD) http://orcid.org/0000-0001-8592-6045

@ marcelozanusso@gmail.com

Fernanda Grill da Silva

(iD) http://orcid.org/0000-0003-2850-2398

@ fernandagrillsilva@gmail.com

Andressa da Silva Arduim

(iD http://orcid.org/0000-0001-8734-6239

Q 2 andressa.arduim@hotmail.com

Daiana Carvalho Borges

(iD) https://orcid.org/0000-0002-0231-9491

@ daianacbrh@gmail.com

Mariângela da Rosa Afonso

(iD https://orcid.org/0000-0002-8853-719X

@ mrafonso.ufpel@gmail.com

Fernanda de Souza-Teixeira

(iD http://orcid.org/0000-0001-7819-9142

@ fsout@unileon.es 